Article

\title{
Frequency, Treatment and Outcome of Immune-Related Toxicities in Patients with Immune-Checkpoint Inhibitors for Advanced Melanoma: Results from an Institutional Database Analysis
}

\author{
Florentia Dimitriou ${ }^{1,2,+}$, Ramon Staeger ${ }^{1,2,+} \mathbb{D}$, Melike Ak ${ }^{1,2,+}$, Matias Maissen ${ }^{2}$, Ken Kudura ${ }^{3}$,

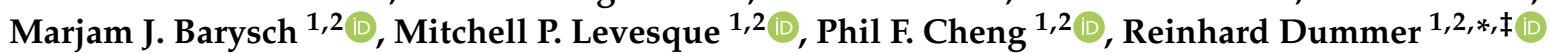 \\ and Joanna Mangana 1,2,
}

1 Department of Dermatology, University Hospital of Zurich, 8091 Zurich, Switzerland; florentia.dimitriou@usz.ch (F.D.); ramon.staeger@usz.ch (R.S.); melike.ak@usz.ch (M.A.); marjam.barysch-bonderer@usz.ch (M.J.B.); mitchell.levesque@usz.ch (M.P.L.); phil.cheng@usz.ch (P.F.C.); johanna.mangana@usz.ch (J.M.)

2 Faculty of Medicine, University of Zurich, 8006 Zurich, Switzerland; matias.maissen@uzh.ch

3 Department of Nuclear Medicine, University Hospital Zurich, 8091 Zurich, Switzerland; ken.kudura@usz.ch

* Correspondence: Reinhard.Dummer@usz.ch; Tel.: +41-44-255-11-11

+ Equally contributed as joint first authors.

$\ddagger$ Equally contributed as last authors.

Citation: Dimitriou, F.; Staeger, R.; Ak, M.; Maissen, M.; Kudura, K.; Barysch, M.J.; Levesque, M.P.; Cheng, P.F.; Dummer, R.; Mangana, J. Frequency, Treatment and Outcome of Immune-Related Toxicities in Patients with Immune-Checkpoint Inhibitors for Advanced Melanoma: Results from an Institutional Database Analysis. Cancers 2021, 13, 2931. https://doi.org/10.3390/ cancers13122931

Academic Editor: Maxime Battistella

Received: 11 May 2021

Accepted: 9 June 2021

Published: 11 June 2021

Publisher's Note: MDPI stays neutral with regard to jurisdictional claims in published maps and institutional affiliations.

Copyright: () 2021 by the authors. Licensee MDPI, Basel, Switzerland. This article is an open access article distributed under the terms and conditions of the Creative Commons Attribution (CC BY) license (https:// creativecommons.org/licenses/by/ $4.0 /)$.
Simple Summary: In this study, we investigated the impact of immune-related adverse events (irAEs) on the survival of advanced melanoma patients treated with immune-checkpoint inhibitors, as well as the effect of corticosteroids and other immune-modulators on clinical outcome. We summarized the kinetics, onset, and outcome of immune-related adverse events (irAEs) in both adjuvant and non-adjuvant settings and we correlated their onset with disease outcome.

\begin{abstract}
Immune checkpoint inhibitors (ICIs) can induce immune-related adverse events (irAEs), which may result in treatment discontinuation. We sought to describe the onset, frequency, and kinetics of irAEs in melanoma patients in a real-life setting and to further investigate the prognostic role of irAEs in treatment outcomes. In this retrospective single-center cohort study, we included 249 melanoma patients. Onset, grade, and resolution of irAEs and their treatment were analyzed. A total of $191(74.6 \%)$ patients in the non-adjuvant and $65(25.3 \%)$ in the adjuvant treatment setting were identified. In the non-adjuvant setting, 29 patients (59.2\%) with anti-CTLA4, 43 (58.1\%) with anti-PD1, and 54 (79.4\%) with anti-PD1/anti-CTLA4 experienced some grade of irAE and these had an improved outcome. In the adjuvant setting, the frequency of irAEs was $84.6 \%$ in anti-CTLA4 and $63.5 \%$ in anti-PD1, but no correlation with disease relapse was observed. Patients with underlying autoimmune conditions have a risk of disease exacerbation. Immunomodulatory agents had no impact on treatment efficacy. IrAEs are correlated with increased treatment efficacy in the nonadjuvant setting. Application of steroids and immunomodulatory agents, such as anti-TNF-alpha or anti-IL6, did not affect ICI efficacy. These data support irAEs as possible prognostic markers for ICI treatment.
\end{abstract}

Keywords: immune-related adverse events; melanoma; immunotherapy; infliximab; tocilizumab

\section{Introduction}

Immune checkpoint inhibitors (ICIs) such as anti-PD1 (programmed cell death 1) and anti-CTLA4 (cytotoxic T lymphocyte antigen 4) antibodies have revolutionized the therapeutic landscape of metastatic melanoma and are approved as first-line therapies in the advanced as well as the adjuvant setting [1-4]. However, the resulting disinhibition of T-cell responses 
can lead to a spectrum of immune-related adverse events (irAEs) that may potentially involve any organ, but most commonly affect the skin, gastrointestinal tract, endocrine glands, liver, lung, and musculoskeletal system [5]. Immune-related AEs are usually manageable, yet in certain cases they can lead to ICI discontinuation or rarely, be fatal $[6,7]$. In patients treated with combined anti-CTLA4/anti-PD1 (ipilimumab and nivolumab), irAEs of any grade can be observed with a frequency of up to $90 \%$ and may lead to treatment discontinuation in up to $50 \%$ of patients [8]. Monotherapies have a better safety profile; interestingly, the frequency and severity of irAEs differ with a higher incidence and grade in anti-CTLA4 than in anti-PD1 regimens [1].

In the treatment of the vast majority of irAEs, corticosteroids are the standard of care. In cases of steroid-refractory irAEs like colitis, pneumonitis, arthritis, and hepatitis, other immunomodulatory treatments such as blocking antibodies against tumor necrosis factor alpha (TNF- $\alpha$ ) or interleukin 6 (IL6) were shown to be effective [5,9-11]. It remains unclear whether these interventions have a negative impact on the antitumor immune response. A pooled analysis of data from the CheckMate 069 and CheckMate 067 clinical trials showed that corticosteroids do not appear to inhibit tumor response [12]. However, high-dose steroids for long durations during anti-PD1 therapy may be associated with poorer survival outcomes [13].

Currently, there are conflicting reports on the prognostic role of irAEs. In some solid tumors, mainly non-small-cell lung cancer (NSCLC), several retrospective studies clearly correlate the occurrence of irAEs under anti-PD1 with an improved response [14,15]; whereas in melanoma, this relationship is less clear [14,16-18].

The aim of this study was to describe the frequency and kinetics of irAEs in melanoma patients treated with ICIs both in unresectable/non-adjuvant stage III/IV and in the adjuvant setting. Furthermore, we investigated the prognostic impact of irAEs on survival and the effect of steroids and other immunomodulators on clinical outcome.

\section{Materials and Methods}

In this single-center retrospective cohort study, we searched the melanoma registry of the Department of Dermatology, University Hospital Zurich, Switzerland, which is comprised of 2766 patients. All adult patients diagnosed with any subtype of melanoma, who received ICI treatment in any line in the adjuvant or unresectable/non-adjuvant setting from February 2011 to May 2020 and with a follow-up period of at least 3 months were included. In patients with multiple ICI treatment lines, the treatment with a confirmed irAE was chosen; if more than one treatment line had confirmed irAEs, then we chose the first ICI treatment; in patients without any toxicities, the first ICI treatment was defined as baseline treatment. The demographical, clinical, and pathological features of eligible patients, including age, sex, primary melanoma origin, stage (according to the American Joint Committee on Cancer 8th edition), and tumor treatment were obtained from our institutional database. Moreover, the electronic medical records of included patients were reviewed for history of autoimmune or auto-inflammatory co-morbidities prior to treatment with ICIs. Date of onset, grade, irAE-directed treatment such as steroids and other immunosuppressive/-modulatory agents, and resolution of irAEs were retrieved. Grading of irAEs was based on the Common Terminology Criteria for Adverse Events (CTCAE), version 5.0 [19].

Overall survival (OS) was calculated from the date of therapy initiation to the last follow-up or death. Progression-free survival (PFS) was calculated from the date of therapy initiation to documented disease progression or last follow-up for non-progressed patients. Three-monthly positron emission tomography-computed tomography (PET-CT) or computed tomography (CT) scans according to the institution protocols were used for the assessment of treatment responses evaluated according to the RECIST criteria [20].

A total of 256 patients were subjected to analysis. Categorical variables were summarized as frequencies. To assess differences in categorical variables, the Fisher's exact test was used. Continuous variables were summarized as median and range. For continuous 
variables, the Wilcoxon rank test was used. Survival differences were assessed with the log-rank test. A $p$-value of $<0.05$ was considered as statistically significant. Time-to-event outcomes (duration of corticosteroids and immunosuppressive agents, time to resolution, OS, PFS) were visualized by Kaplan-Meier (KM) curves. All statistical analyses were performed by using $\mathrm{R}$ version 4.0.1.

\section{Results}

\subsection{Demographics}

A total of 256 patients, 191 (74.6\%) in advanced/non-adjuvant and $65(25.3 \%)$ in adjuvant setting, with median age of 63 years (range 25-92) were identified (Table 1).

Table 1. Patients' characteristics in the overall population, non-adjuvant and adjuvant setting.

\begin{tabular}{|c|c|c|c|c|c|c|c|}
\hline \multirow[b]{2}{*}{ Characteristic } & \multirow[b]{2}{*}{$\begin{array}{c}\text { Overall } \\
\text { Population, } \\
n=256\end{array}$} & \multicolumn{3}{|c|}{ Non-Adjuvant Setting, $n=191$} & \multicolumn{3}{|c|}{ Adjuvant Setting, $n=65$} \\
\hline & & $\begin{array}{c}\text { Patients with } \\
\text { No Toxicities, } \\
\quad n=65\end{array}$ & $\begin{array}{c}\text { Patients with } \\
\text { Toxicities, } n= \\
126\end{array}$ & $p$-Value ${ }^{1}$ & $\begin{array}{c}\text { Patients with } \\
\text { No Toxicities, } \\
\quad n=21\end{array}$ & $\begin{array}{c}\text { Patients with } \\
\text { Toxicities, } n=44\end{array}$ & $p$-Value ${ }^{1}$ \\
\hline $\begin{array}{l}\text { Age at treatment } \\
\text { start (range) }\end{array}$ & $63(25-92)$ & $68(35-89)$ & $61(33-92)$ & 0.07 & $63(31-83)$ & $55(25-87)$ & 0.2 \\
\hline $\begin{array}{l}\text { AJCCv8 Stage at } \\
\text { treatment start, } n \\
(\%)\end{array}$ & & & & 0.8 & & & 0.8 \\
\hline II & $4(1.6 \%)$ & - & - & - & $2(9.5 \%)$ & $2(4.5 \%)$ & \\
\hline III & $65(25 \%)$ & $4(6.2 \%)$ & $10(7.9 \%)$ & & $16(76 \%)$ & $35(80 \%)$ & \\
\hline IV & $187(73 \%)$ & $61(94 \%)$ & $116(92 \%)$ & & $3(14 \%)$ & $7(16 \%)$ & \\
\hline $\begin{array}{c}\text { Melanoma } \\
\text { subtype, } n(\%)\end{array}$ & & & & 0.3 & & & $>0.9$ \\
\hline Cutaneous & $201(79 \%)$ & $49(75 \%)$ & $97(77 \%)$ & & $18(86 \%)$ & $37(84 \%)$ & \\
\hline Acral & $3(1.2 \%)$ & $2(3.1 \%)$ & - & & - & $1(2.3 \%)$ & \\
\hline Mucosal & $14(5.5 \%)$ & $4(6.2 \%)$ & $7(5.6 \%)$ & & $1(4.8 \%)$ & $2(4.5 \%)$ & \\
\hline Unknown primary & $24(9.4 \%)$ & $7(11 \%)$ & $11(8.7 \%)$ & & $2(9.5 \%)$ & $4(9.1 \%)$ & \\
\hline Uveal & $14(5.5 \%)$ & $3(4.6 \%)$ & $11(8.7 \%)$ & & - & - & \\
\hline $\begin{array}{c}\text { Autoimmune } \\
\text { disease in medical } \\
\text { history, } n(\%)\end{array}$ & & & & 0.7 & & & 0.4 \\
\hline Chronic thyroiditis & $5(2.0 \%)$ & $2(3.1 \%)$ & $2(1.6 \%)$ & & $1(4.8 \%)$ & - & \\
\hline Diabetes type 1 & $3(1.2 \%)$ & $1(1.5 \%)$ & $2(1.6 \%)$ & & - & - & \\
\hline $\begin{array}{l}\text { Inflammatory } \\
\text { bowel disease }\end{array}$ & $2(0.8 \%)$ & - & $1(0.8 \%)$ & & - & $1(2.3 \%)$ & \\
\hline $\begin{array}{l}\text { Rheumatoid } \\
\text { arthritis }\end{array}$ & $6(2.3 \%)$ & - & $3(2.4 \%)$ & & - & $3(6.8 \%)$ & \\
\hline Multiple Sclerosis & $1(0.4 \%)$ & - & $1(0.8 \%)$ & & - & - & \\
\hline $\begin{array}{l}\text { Bechterew's } \\
\text { disease }\end{array}$ & $1(0.4 \%)$ & $1(1.5 \%)$ & - & & - & - & \\
\hline Sarcoidosis & $2(0.8 \%)$ & - & $2(1.6 \%)$ & & - & - & \\
\hline $\begin{array}{c}\text { Presence of brain } \\
\text { metastases at } \\
\text { treatment start, } n \\
(\%)\end{array}$ & $48(19 \%)$ & $16(25 \%)$ & $32(25 \%)$ & $>0.9$ & - & - & \\
\hline $\begin{array}{c}\text { Type of treatment, } \\
n(\%)\end{array}$ & & & & 0.01 & & & 0.2 \\
\hline $\begin{array}{l}\text { Anti-PD1/Anti- } \\
\text { CTLA4 }\end{array}$ & $68(27 \%)$ & $14(22 \%)$ & $54(43 \%)$ & & - & - & \\
\hline
\end{tabular}


Table 1. Cont.

\begin{tabular}{|c|c|c|c|c|c|c|c|}
\hline \multirow[b]{2}{*}{ Characteristic } & \multirow[b]{2}{*}{$\begin{array}{c}\text { Overall } \\
\text { Population, } \\
n=256\end{array}$} & \multicolumn{3}{|c|}{ Non-Adjuvant Setting, $n=191$} & \multicolumn{3}{|c|}{ Adjuvant Setting, $n=65$} \\
\hline & & $\begin{array}{c}\text { Patients with } \\
\text { No Toxicities, } \\
\quad n=65\end{array}$ & $\begin{array}{c}\text { Patients with } \\
\text { Toxicities, } n= \\
126\end{array}$ & $p$-Value ${ }^{1}$ & $\begin{array}{c}\text { Patients with } \\
\text { No Toxicities, } \\
\quad n=21\end{array}$ & $\begin{array}{c}\text { Patients with } \\
\text { Toxicities, } n=44\end{array}$ & $p$-Value ${ }^{1}$ \\
\hline Anti-PD1 & $126(49 \%)$ & $31(48 \%)$ & $43(34 \%)$ & & $19(90 \%)$ & $33(75 \%)$ & \\
\hline Anti-CTLA4 & $62(24 \%)$ & $20(31 \%)$ & $29(23 \%)$ & & $2(9.5 \%)$ & $11(25 \%)$ & \\
\hline $\begin{array}{c}\text { Reason for } \\
\text { treatment } \\
\text { discontinuation, } n \\
(\%)\end{array}$ & & & & & & & 0.01 \\
\hline Toxicity & $35(13.7 \%)$ & - & $25(20 \%)$ & & - & $10(23 \%)$ & \\
\hline $\begin{array}{l}\text { Disease progres- } \\
\text { sion/recurrence }\end{array}$ & $112(43.8 \%)$ & $51(79 \%)$ & $46(71 \%)$ & & $3(14 \%)$ & $12(27 \%)$ & \\
\hline $\begin{array}{l}\text { Treatment } \\
\text { completed }\end{array}$ & $40(15.6 \%)$ & $1(1.5 \%)$ & $4(3.2 \%)$ & & $16(76 \%)$ & $19(43.1 \%)$ & \\
\hline $\mathrm{CR}$ & $34(13.3 \%)$ & $7(11 \%)$ & $27(21 \%)$ & & - & - & \\
\hline $\begin{array}{l}\text { Patient's/Investigator's } \\
\text { choice }\end{array}$ & $22(8.6 \%)$ & $5(7.7 \%)$ & $12(9.5 \%)$ & & $2(9.5 \%)$ & $3(6.8 \%)$ & \\
\hline
\end{tabular}

Best Overall

Response (BOR),

$n(\%)$

\begin{tabular}{ccccc}
\hline CR & $95(40 \%)$ & $8(12 \%)$ & $44(37 \%)$ & - \\
\hline PR & $36(15 \%)$ & $12(19 \%)$ & $24(20 \%)$ & - \\
\hline SD & $12(5.0 \%)$ & $2(3.1 \%)$ & $10(8.4 \%)$ & - \\
\hline PD & $95(40 \%)$ & $42(66 \%)$ & $41(34 \%)$ & - \\
\hline
\end{tabular}

${ }^{1}$ Wilcoxon rank sum test; Fisher's exact test; Pearson's Chi-squared test; Abbreviations: CR; complete response, PR; partial response, SD; stable disease, $\mathrm{PD}$; progressive disease.

Of these, 170 patients $(66.4 \%)$ experienced some grade (1-4, CTCAEv5) of toxicity. The majority of the primary tumors in the overall study cohort were cutaneous $(n=201$, $79 \%)$ and unknown primary $(n=24,9.4 \%)$, and most of the patients had stage IV disease ( $n=187,73 \%)$. The vast majority of the patients $(n=186,73 \%)$ were treatment naïve, whereas 27 patients $(11 \%)$ had previously immunotherapy and 24 patients $(9.4 \%)$ targeted therapy. Systemic treatments included anti-PD1/anti-CTLA4 in $68(27 \%)$ patients, singleagent anti-PD1 in $126(49 \%)$ patients and anti-CTLA4 in $62(24 \%)$ patients. Median follow up (FU) was 27 months (range 3-108) and at the end of the observation period, 92 (36\%) patients died due to melanoma, one $(0.4 \%)$ patient died from adverse events and seven (3\%) patients died of other causes, while the rest of $156(61 \%)$ patients were still alive.

\subsection{Adverse Events}

In the overall study population, treatment-related toxicities were reported in $11(84.6 \%)$ patients with anti-CTLA4 and in 33 patients $(63.5 \%)$ patients with anti-PD1 in the adjuvant setting. A total of 29 patients $(59.2 \%)$ with anti-CTLA4, 43 (58.1\%) patients with antiPD1 and $54(79.4 \%)$ patients with anti-PD1/anti-CTLA4 in the non-adjuvant setting had treatment-related toxicities (Figure 1A). Median time to onset of irAEs was 52 days (range $0-1328)$. Overall, the most common treatment-related toxicities included thyroiditis (29.4\%), colitis $(27.6 \%)$, rash $(24.7 \%)$, hepatitis $(18.2 \%)$, arthritis $(13.5 \%)$, hypophysitis $(12.9 \%)$, pneumonitis (8.8\%), vitiligo-like depigmentation (8.8\%) and pancreatitis (7.6\%). Other adverse events of special interest included myocarditis (3.5\%), cytokine release syndrome (CRS, $1.8 \%)$, type 1 diabetes $(1.2 \%)$, uveitis $(1.2 \%)$, encephalitis $(0.6 \%)$, meningitis $(0.6 \%)$, myositis $(0.6 \%)$ and nephritis $(0.6 \%)$. In the non-adjuvant setting, combined immunotherapy was complicated with severe (grade $\geq 3$ ) toxicities in $58.8 \%$ of the cases, compared to $22.4 \%$ and $13.3 \%$ in anti-CTLA4 and anti-PD1 agents, respectively. In the adjuvant setting, grade 3 
and higher toxicities were reported in $38.4 \%$ and $13.5 \%$ of patients treated with anti-CTLA4 and anti-PD1, respectively (Figure 1B).

A

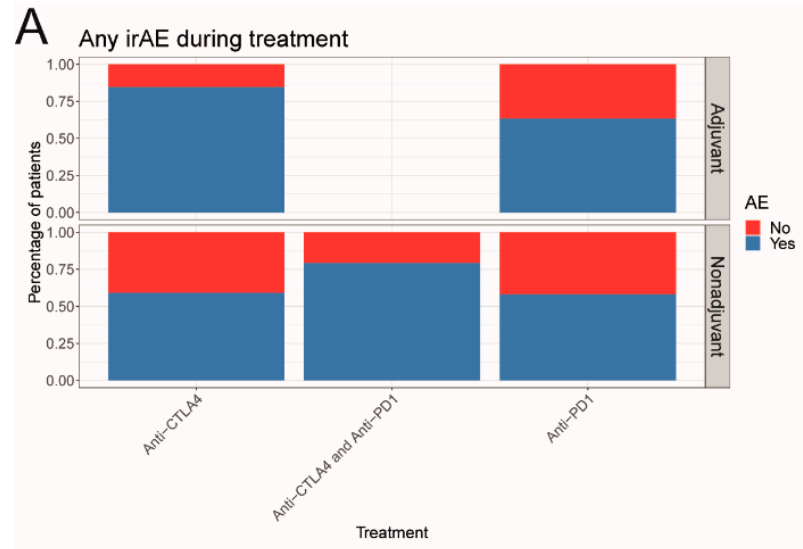

C

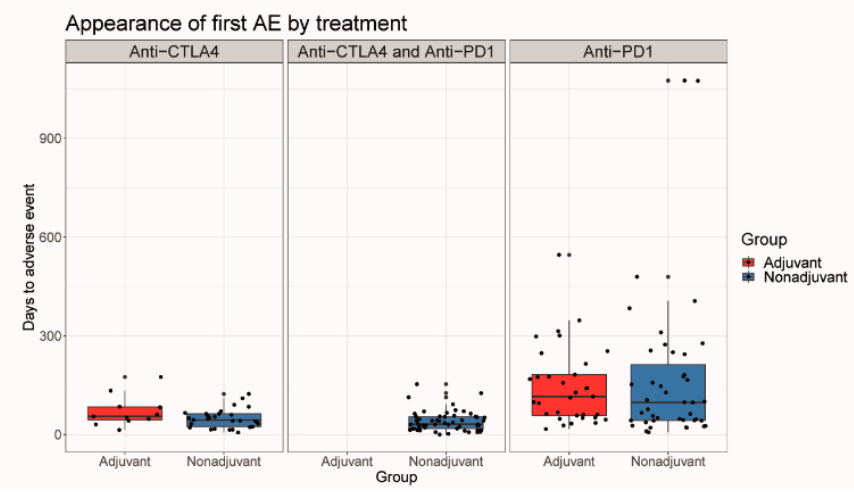

B

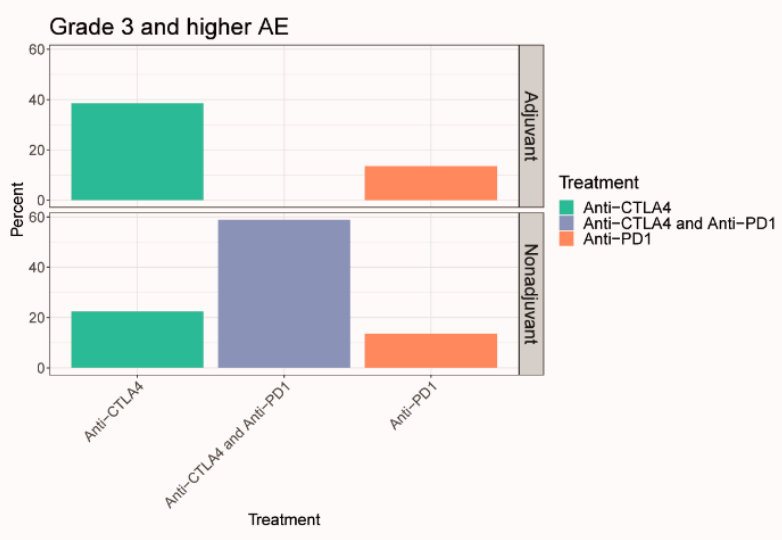

Figure 1. Frequency and time of appearance of irAEs in the adjuvant and non-adjuvant setting. (A) Barplot representing the frequencies of any irAE by treatment faceted by the adjuvant and non-adjuvant setting. (B) Barplot representing the frequencies of grade 3 and higher irAE by treatment faceted by the adjuvant and non-adjuvant setting. (C) Boxplots representing the appearance of the first irAE by treatment in the adjuvant and non-adjuvant setting.

Late-onset toxicities, i.e., toxicities that were observed after treatment discontinuation, were reported in nine patients, with median time to onset 132 days (range 100-334) after treatment discontinuation and included most frequently endocrinological toxicities $(n=4$, including hypophysitis, thyroiditis and type 1 diabetes), skin toxicities $(n=3)$, pneumonitis $(n=1)$ and inflammatory/CRS symptoms $(n=1)$. When stratified according to the treatment regimen, anti-PD1 treatment was complicated more frequently with immune-related arthritis, pneumonitis, myocarditis, thyroiditis and hematologic toxicities, whereas antiCTLA4-driven toxicities included colitis, hypophysitis and skin rash; types of toxicities and kinetics according to treatment are summarized in Figure 2.

\subsection{Adverse Events in Non-Adjuvant/Unresectable Setting}

In the non-adjuvant/unresectable setting (AJCCv8 Stage III-IV), treatment-related toxicities were reported in 126 patients $(65.6 \%)$, of which $45 \%$ experienced $\geq 2$ toxicities (range 2-7) (Table 1). A total of $74(39 \%), 68(36 \%)$, and $49(26 \%)$ patients were treated with anti-PD1, anti-CTLA4/anti-PD1 and anti-CTLA4 respectively. Treatment-related toxicities led to treatment discontinuation in 25 patients (20\%), mostly in the combined immunotherapy group. Median time to toxicity onset was 43.5 days (range 0-1328); 31.5 days for anti-PD1/anti-CTLA4, 44 days for anti-CTLA4 and 97 days for anti-PD1 (Figure 1C). The most frequent toxicities for combined immunotherapy included immunerelated thyroiditis $(30.9 \%)$, hepatitis $(29.4 \%)$ and colitis $(26.5 \%)$. In the monotherapy regimens, anti-PD1 treatment was mostly associated with skin (vitiligo-like depigmentation 
12.2\%, rash 9.5\%) and endocrinological (thyroiditis 19\%, hypophysitis $9.5 \%$ ) toxicities, whereas colitis $(34.7 \%)$ and skin rash $(22.4 \%)$ were commonly observed in single-agent anti-CTLA4 (Figure 3A).

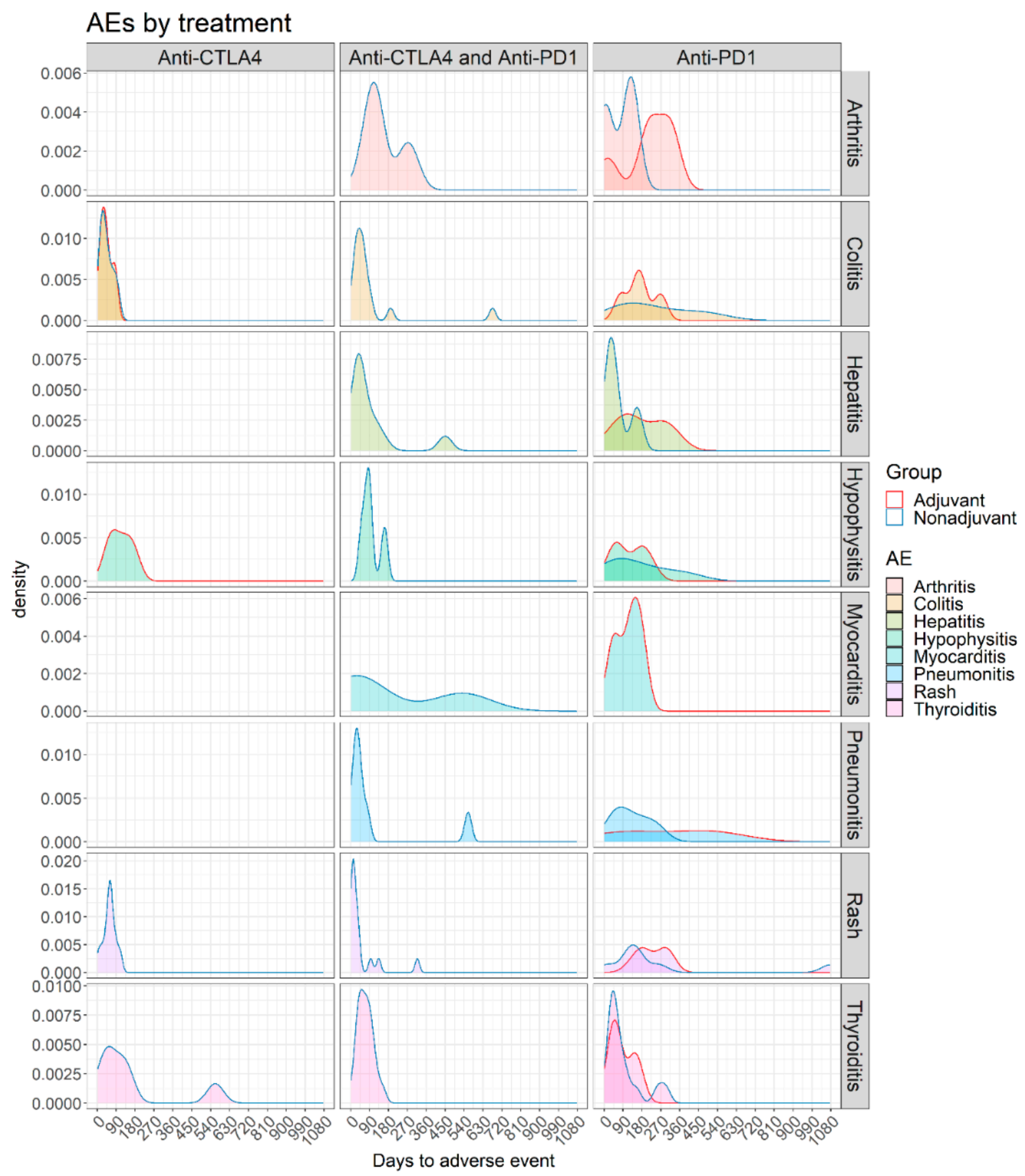

Figure 2. Density plots representing the time of appearance of arthritis, colitis, hepatitis, hypophysitis, myocarditis, pneumonitis, rash and thyroiditis in adjuvant and non-adjuvant patients treated with anti-CTLA4, anti-PD1, and antiCTLA4 with anti-PD1.

Treatment discontinuation was most commonly related to combined immunotherapy, with similar patterns as in the general study population. Of note, immune-related arthritis led to treatment discontinuation in $8.8 \%$ of patients with combined immunotherapy, as 
well as $9.5 \%$ in single-agent anti-PD1. Similar to combination treatment, colitis was the most frequent event that led to treatment discontinuation in anti-CTLA4 alone $(18.4 \%$ and $14.7 \%$, respectively).

\subsection{Adverse Events in Adjuvant Setting}

Out of the 65 patients that were treated in the adjuvant setting (AJCCv8, Stage II-IV), $44(67.7 \%)$ patients presented with treatment-related adverse events, prompting treatment discontinuation in $10(23 \%)$ patients (Table 1$)$. The selected treatment regimen was antiPD1 alone in $33(75 \%)$ patients, whereas $11(25 \%)$ patients were treated with adjuvant anti-CTLA4, in a reduced adjuvant dose (3mg/kg Q3W). Sixteen (36.4\%) patients had multiple toxicities (range 2-5). Median time to toxicity onset was 89.5 days (range 14-546); 115 days for anti-PD1 and 55 days for anti-CTLA4 (Figure 1C). In the single-agent anti-PD1, the toxicity pattern was similar to the non-adjuvant setting, with increasing frequency of hepatitis $(9.6 \%)$, arthritis $(9.6 \%)$ and colitis $(7.7 \%)$ (Figure 3B).

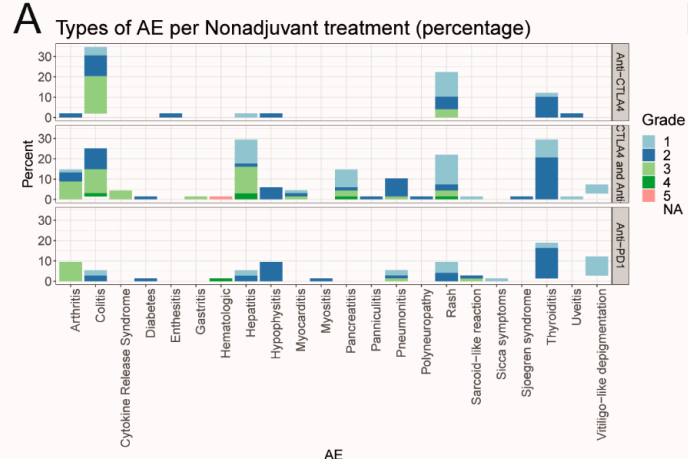

C

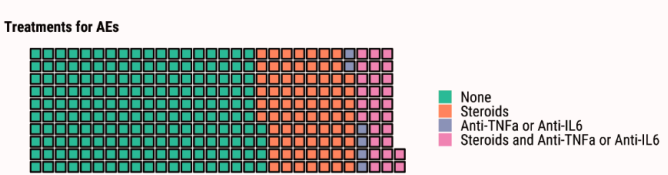

B
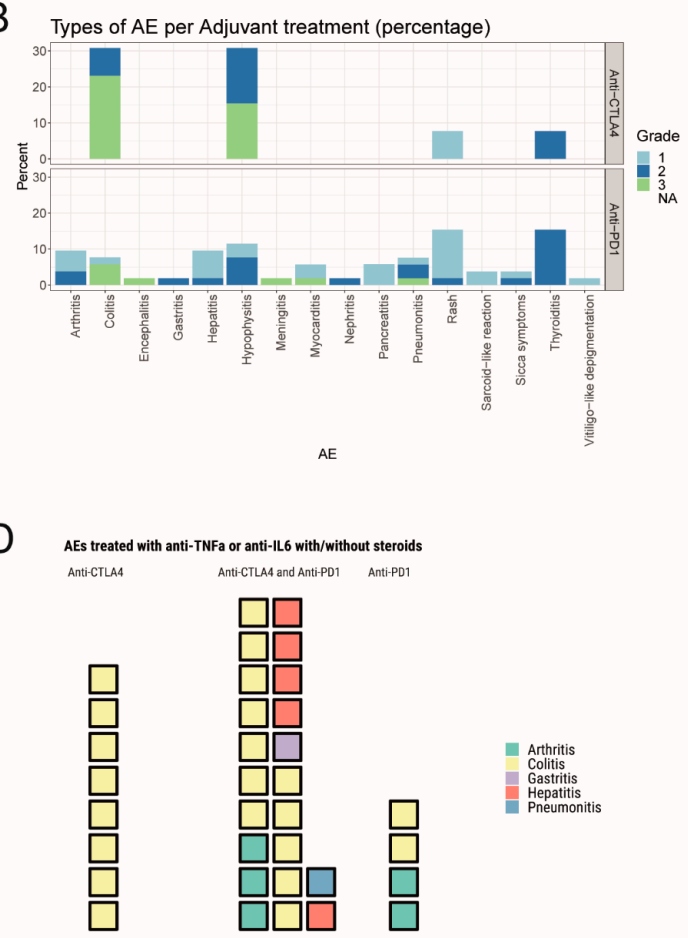

Figure 3. Types/grades of irAEs and their treatments in the adjuvant and non-adjuvant setting. (A) Stacked barplots representing the frequency of each time of irAE for anti-CTLA4, anti-PD1, and anti-CTLA4 with anti-PD1 in the nonadjuvant setting. (B) Stacked barplots representing the frequency of each time of irAE for anti-CTLA4 and anti-PD1 in the adjuvant setting. (C) Waffle plot showing the 292 irAEs untreated, treated with steroids, treated with anti-TNF $\alpha$ or anti-IL6, and treated with steroids and anti-TNF $\alpha$ or anti-IL6. (D) Waffle plot showing the different irAEs treated with anti-TNF $\alpha$ or anti-IL6 with and without steroids for anti-CTLA4, anti-PD1, and anti-CTLA4 with anti-PD1.

Nevertheless, and compared to the non-adjuvant group, toxicities with long-term effects, or potentially fatal effects were more commonly observed and included endocrinological toxicities (thyroiditis $15.4 \%$, hypophysitis $11.5 \%$ ), myocarditis $(5.7 \%)$, meningitis $(1.9 \%)$ and encephalitis $(1.9 \%)$. Immune-related colitis $(5.8 \%)$, encephalitis $(1.9 \%)$, meningitis $(1.9 \%)$, myocarditis $(1.9 \%)$ and pneumonitis $(1.9 \%)$ were the grade $3-4$ toxicities that led to treatment discontinuation in this group.

Similar to the non-adjuvant group, in the single-agent anti-CTLA4, colitis (30.8\%) and hypophysitis (30.8\%) were frequent treatment-related toxicities that were associated with severe, grade $3-4$ adverse events in $23.1 \%$ and $15.4 \%$ of the cases, respectively. 


\subsection{Immunomodulatory Agents}

Out of the 292 irAEs from 170 patients, 74 events were treated with steroids alone, 28 with steroids and an immunomodulatory treatment, including either anti-TNF $\alpha(n=25)$ or anti-IL6 $(n=2)$ or both $(n=1)$. Patients received for six events anti-TNF $\alpha$ alone, and 184 received neither steroids nor an immunomodulatory treatment (Figure 3C). Within the 34 cases that received an immunomodulatory agent, the vast majority $(23 / 34)$ of the toxicities were associated with anti-CTLA4/anti-PD1 and included colitis grade $\geq 2$ $(n=13)$, hepatitis grade $\geq 3(n=5)$, arthritis grade $\geq 2(n=3)$, gastritis grade $3(n=1)$ and pneumonitis grade $2(n=1)$. Other toxicities included arthritis grade $3(n=2)$ and colitis grade $2(n=1)$, associated with single-agent anti-PD1 and colitis grade $\geq 2(n=8)$, associated with anti-CTLA4 (Figure 3D). Anti-TNF- $\alpha$ was preferred as the immunomodulatory agent in most colitis cases (21/22) and treatment benefit was observed in 20/21 cases; one patient with grade 3 colitis was treated with first-line anti-TNF- $\alpha$ as a steroid-sparing option with toxicity resolution. Of note, no benefit was observed in two cases of grade 3 hepatitis and grade 3 colitis treated with anti-TNF- $\alpha$, thus requiring further immunosuppression. Anti-IL6 was administered in one case of pneumonitis grade 2 and colitis grade 3 , with toxicity resolution. A patient with colitis grade 3 , who did not respond to either steroids or anti-TNF- $\alpha$, was treated with anti-IL6, with event resolution.

\subsection{Patients with Underlying Autoimmune/Auto-Inflammatory Comorbidities}

Twenty $(7.8 \%)$ patients with underlying autoimmune or auto-inflammatory conditions at baseline, including inflammatory bowel disease (IBD, $n=3)$, type 1 diabetes $(n=3)$, Bechterew's disease $(n=1)$, multiple sclerosis (MS, $n=1)$, rheumatoid arthritis (RA, $n=6$ ), chronic thyroiditis $(n=4)$ and sarcoidosis $(n=2)$ were identified (Table 1). At the time of immunotherapy initiation, seven (35\%) patients had an inactive underlying disease, and five $(25 \%)$ required symptomatic treatment or substitution only. Low-dose steroids or systemic immunosuppressive treatment was present in six (30\%) cases. One patient with MS was on fingolimod treatment, which was discontinued prior to immunotherapy (anti-PD1/anti-CTLA4), without MS exacerbation during treatment. Notably, prophylactic treatment with anti-TNF- $\alpha$ was administered in a patient with inactive IBD, subsequently treated with anti-PD1, without an IBD flare.

The majority of the patients received anti-PD1 alone $(n=11)$; nevertheless, six patients were treated with anti-PD1/anti-CTLA4 and three with anti-CTLA4. Five (25\%) patients tolerated the systemic treatment well, with neither treatment-related toxicities nor disease flare, whereas fifteen (75\%) patients, four from the adjuvant and eleven from the advanced/unresectable setting, developed treatment-related toxicities and seven (35\%) patients had exacerbation of their underlying disease. Disease flare $(n=4)$ of inactive conditions or worsening $(n=3)$ was noted in rheumatoid arthritis $(n=2)$, chronic thyroiditis $(n=3), \operatorname{IBD}(n=1)$ and type 1 diabetes $(n=1)$.

\subsection{Efficacy}

Patients that presented with irAEs in the adjuvant setting showed no superior prognosis with regard to relapse-free survival (RFS) compared to the patients without irAEs, in the different treatment regimens ( $p=0.43$ for anti-CTLA4 and $p=0.65$ for anti-PD1, Figure 4A). Overall survival (OS) was similar between the two groups (Figure 4B), but mOS was not reached. Univariate Cox regression analysis for the clinical features (age, sex and presence of irAEs) showed no significant results for RFS and OS (Figure S1).

In the non-adjuvant setting, patients with irAEs presented with more favorable PFS compared to non-irAEs in all treatment regimens ( $p=0.0036$ for anti-CTLA4, $p=0.0022$ for anti-PD1 and $p<0.0001$ for anti-CTLA4/anti-PD1, (Figure 4C), which was also translated to survival benefit on OS rates except for anti-PD1 ( $p=0.22$, Figure 4D). Univariate Cox regression analysis for the clinical features (age, sex, elevated LDH at treatment start and presence of irAEs and brain metastases) showed that presence of irAEs was associated with prolonged PFS in anti-CTLA4 (HR 0.34, $p=0.007$ ), anti-PD1 (HR 0.32, $p=0.002$ ) and 
anti-PD1/anti-CTLA4 (HR 0.08, $p<0.001$ ) (Figure S2). Male sex negatively influenced PFS in anti-CTLA4 treatment (HR 2.84, $p=0.024)$. Elevated LDH at treatment start was associated with poor OS in anti-CTLA4 (HR 8.04, $p<0.001$ ), anti-PD1 (HR 2.67, $p=0.034$ ) and anti-PD1/anti-CTLA4 (HR 0.16, $p<0.001$ ). The presence of irAEs was significant for prolonged OS in anti-CTLA4 (HR 0.34, $p=0.026$ ) and anti-PD1 / anti-CTLA4 (HR 0.16, $p<0.001$.

Patients that experienced an irAE were assessed for whether no treatment or treatment with steroids with or without an immunomodulatory agent affected PFS and OS. Of note, steroids and other anti-inflammatory drugs had no significant impact on progressionfree survival or overall survival in the adjuvant and non-adjuvant setting (PFS and OS, Figure 5A-D).

A
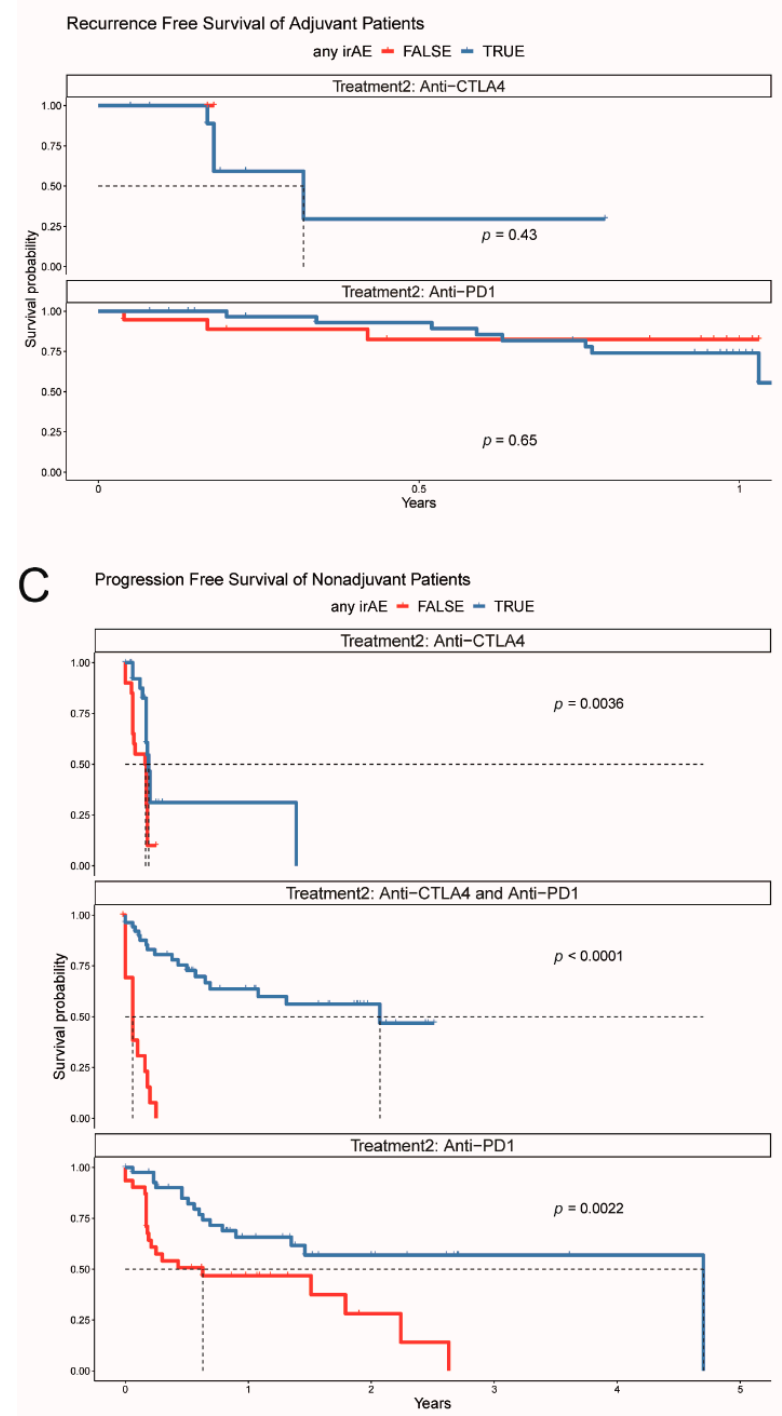

B

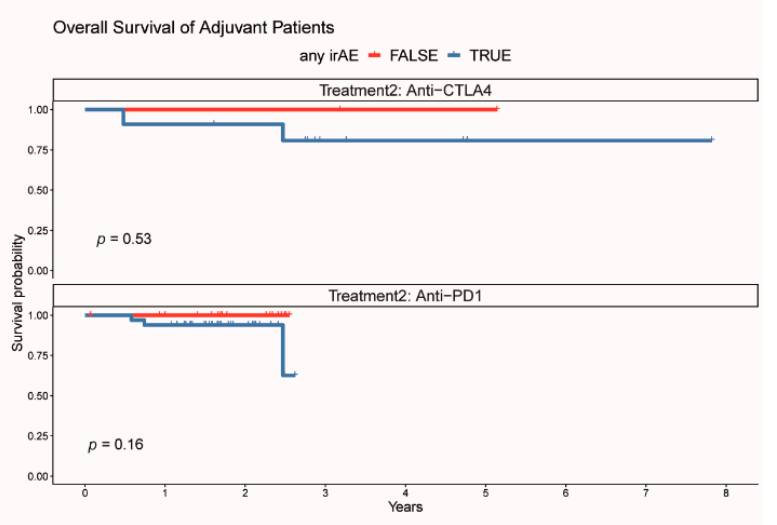

Overall Survival of Nonadjuvant Patients

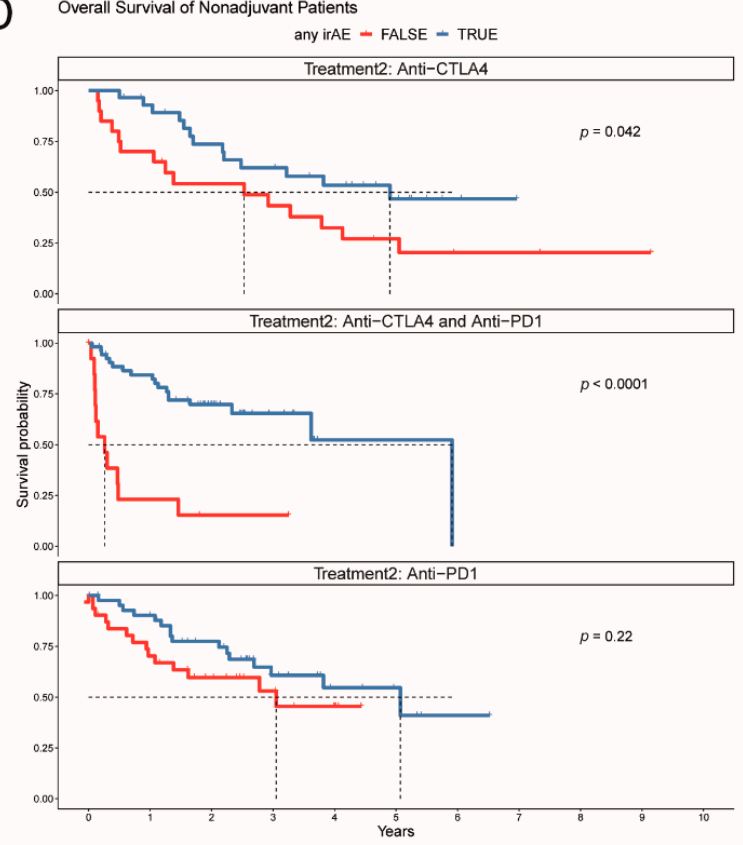

Figure 4. Kaplan-Meier plots for the adjuvant and non-adjuvant setting stratified by the appearance of irAE. (A) Recurrencefree survival for anti-CTLA4 and anti-PD1 in the adjuvant setting. (B) Overall survival for anti-CTLA4 and anti-PD1 in the adjuvant setting. (C) Progression-free survival for anti-CTLA4, anti-PD1, and anti-CTLA4 with anti-PD1 in the non-adjuvant setting. (D) Overall survival for anti-CTLA4, anti-PD1, and anti-CTLA4 with anti-PD1 in the non-adjuvant setting. 
A

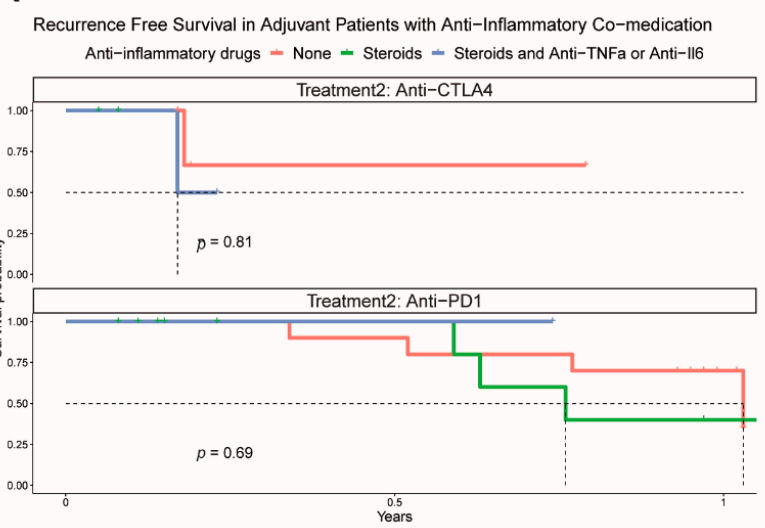

$C_{\text {Progression Free Survival in Nonadjuvant Patients with Anti-Inflammatory Co-medication }}$ Anti-inflammatory drugs - None - Steroids - Steroids and Anti-TNFa or Anti-116

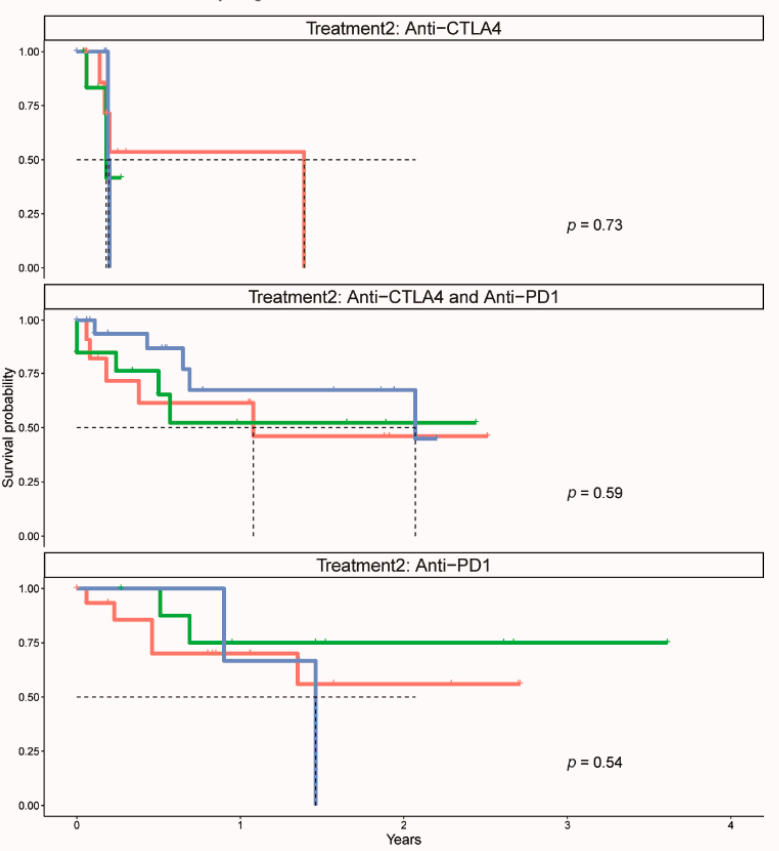

B

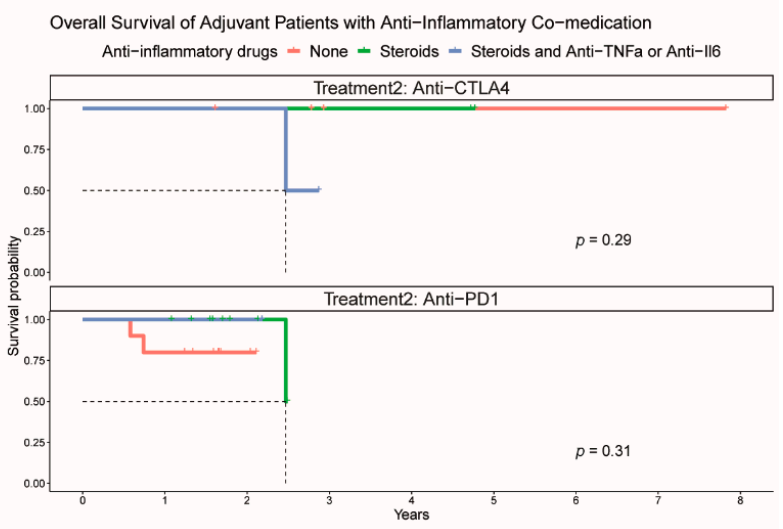

D Overall Survival in Nonadjuvant Patients with Anti-Inflammatory Co-medication Anti-inflammatory drugs - None - Steroids - Steroids and Anti-TNFa or Anti-II6
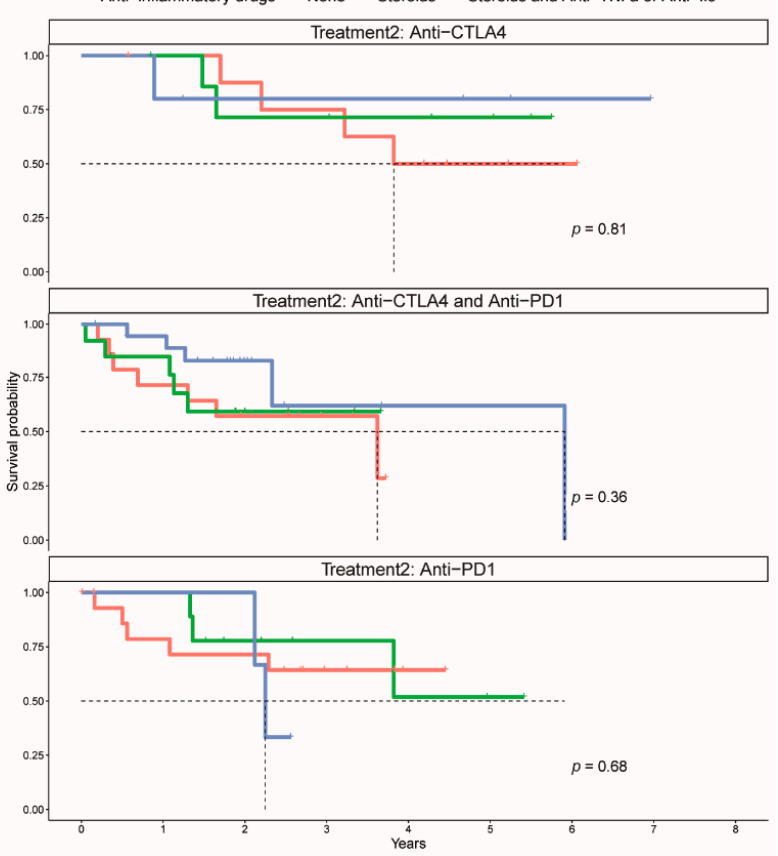

Figure 5. Kaplan-Meier plots for the adjuvant and non-adjuvant settings stratified by treatment for the irAE. (A) Recurrencefree survival in the adjuvant setting for anti-CTLA4 and anti-PD1. (B) Overall survival in the adjuvant setting for anti-CTLA4 and anti-PD1. (C) Progression-free survival in the non-adjuvant setting for anti-CTLA4, anti-PD1, and anti-CTLA4 with anti-PD1. (D) Overall survival in the non-adjuvant setting for anti-CTLA4, anti-PD1, and anti-CTLA4 with anti-PD1.

\section{Discussion}

Although ICIs are currently in widespread use in oncology and their efficacy in melanoma patients is unquestionable, there is contradictory evidence on the correlation of irAEs with clinical outcome. In this retrospective study, patients with irAEs showed improved PFS in all unresectable/non-adjuvant treatment regimens, compared to patients without irAEs. In combined immunotherapy with anti-PD1/anti-CTLA4, the presence of irAEs was associated with an OS benefit as well. As such, irAEs may represent an indicator of treatment efficacy in these patients. Of note, in the adjuvant setting, no correlation of irAEs and relapse-free or OS was observed. Organ-specific irAEs differed between the two agents; anti-PD1 treatment was complicated more frequently with arthritis, pneumonitis, myocarditis, thyroiditis and hematologic toxicities, whereas anti-CTLA4driven toxicities included colitis, hypophysitis and skin rash. Treatment of irAEs with steroids and other immunomodulatory agents, such as anti-TNF $\alpha$ and anti-IL6, had no impact on treatment efficacy. 
Our results are in line with several retrospective studies suggesting significant benefit towards tumor control and survival in immunotherapy-treated patients that experience any kind of irAE, with the majority of reports coming from non-small-cell lung cancer field (NSCLC) [14,15,21,22]. However, for melanoma, the association between toxicity and efficacy is not as linear as observed in other cancer types. The majority of published studies have indicated either a confirmed PFS or OS benefit for dermatologic toxicities (especially for hypopigmentation) or no benefit at all; a retrospective study of melanoma patients treated with anti-PD1 reported OS benefit in patients with irAEs but in the multivariate analysis only $\geq$ grade 3 toxicities remained a significant confounder for OS [23,24]. A pooled analysis of the Keynote 001, 002 and 006 clinical trials in advanced melanoma patients treated with pembrolizumab demonstrated no efficacy difference in patients with irAEs compared to no irAE at the landmark analysis of 21 weeks. Still, the analysis was conducted only in $29.5 \%$ of the total population (463/1567 patients) including only those that did not progress until week 21, therefore excluding both patients with innate early resistance or those with late toxicities [18].

Notably, the frequency and type of irAE differed significantly in the adjuvant setting. This was particularly prominent in anti-PD1 treatment and in all grades of irAEs, but frequency of $\geq$ grade 3 irAEs was similar between the adjuvant and non-adjuvant setting. This observation is pertinent for risk/benefit assessment in adjuvant treatment, as many patients may already be cured by surgery alone. Furthermore, we observed a higher incidence of toxicities with long-term effects, or potentially fatal toxicities in the adjuvant setting with an increased frequency of myocarditis $(6.7 \%)$, meningitis $(2.2 \%)$, encephalitis $(2.2 \%)$ and endocrinological toxicities (thyroiditis $17.8 \%$ and hypophysitis $13.3 \%)$. In fact, irAEs with long-term or potentially fatal effects were more commonly observed than in the non-adjuvant setting and this risk should not be neglected in adjuvant treatment decision in resected melanoma [25]. The difference in frequency of irAEs might be attributed to the treatment exposure time and the patient's intrinsic factors, as well as immune status in stage III compared to advanced, unresectable melanoma [26]. In contrast to a recent report of the EORTC-1325/Keynote-054 study in patients with resected Stage III melanoma, where the presence of endocrine and skin toxicities in the pembrolizumab arm was correlated with improved RFS rates, our study did not confirm this superiority in risk of progression in the adjuvant setting [17]. However, the small number of patients in this setting precludes any statistical conclusion. Interestingly and besides the appearance of vitiligo-like depigmentation under interferons [27], no other reports with anti-PD1 or anti-CTLA4 monotherapy have been published so far confirming the prognostic value of irAEs in the adjuvant setting either prospectively or retrospectively.

In our cohort, $66.4 \%$ experienced at least one irAE; $79 \%$ with anti-PD1/anti-CTLA4, $60 \%$ with anti-PD1 and $64 \%$ with anti-CTLA 4 alone, which is in the expected range, compared to data from randomized clinical trials. Notably, median time to toxicity onset was longer in the adjuvant setting versus non-adjuvant ( 89.5 versus 43.5 days). This is probably treatment-regimen-associated since more than $2 / 3$ of the patients in the non-adjuvant setting received an anti-CTLA4-directed treatment, either alone or in combination with anti-PD1. Besides, nivolumab was initially tested at $3 \mathrm{mg} / \mathrm{kg}$-weekly in the CheckMate238 trial [4] and nivolumab/ipilimumab are administered at a dose of 1 and $3 \mathrm{mg} / \mathrm{kg}$ 3-weekly at the induction phase. Nivolumab, which is the treatment regimen preferred in the adjuvant setting, is now also approved and administered as a flat dose of $480 \mathrm{mg}$ 4-weekly. While it is likely that such alterations in dosing and schedule do not impact efficacy or toxicity, the optimal treatment duration is not known and data from alternative dosing schedules from anti-CTLA4 should be thoroughly reviewed. In the adjuvant setting, ipilimumab is approved at a dose of $10 \mathrm{mg} / \mathrm{kg}$, but its use is limited due to high toxicity. As such, we applied adjuvant ipilimumab in the conventional dose of $3 \mathrm{mg} / \mathrm{kg}$ 3-weekly, with significantly improved tolerability [28]. Although in the Checkmate-511 trial the induction dose of anti-CTLA4 does not seem to affect efficacy [29], in the Checkmate-915 
trial, low-dose ipilimumab combined with nivolumab was not superior to nivolumab alone [30].

Our data suggest that steroids for irAEs or other immunomodulating agents for steroid-refractory toxicities do not have a direct impact on prognosis. Similar results have been reported in other studies and in a pooled analysis of pembrolizumab with patients that received steroids for irAEs experiencing similar efficacy outcomes with those without $[12,18,31]$. However, the impact of duration of the immunosuppressive treatment on disease outcome was not addressed in this study. In a secondary analysis of the EORTC-1325/Keynote-054 study, treatment effect of adjuvant pembrolizumab after 30 days of systemic steroid use appeared to be lower than without steroid use or by day 30 of systemic steroid use [17]. In another recent multicenter observational study on concomitant medications and outcomes in patients with solid tumors treated with ICIs, patients with steroids at baseline for cancer-related indications had significantly worse PFS and OS than those without or those with steroids for non-cancer-related indications [32]. Nevertheless, the authors did not include patients with irAEs requiring steroids in the non-cancer-related indications group. Taken all together, we assume that steroids given for irAEs for a short time or those given for symptom control have different effects on survival and outcome with the first neutralizing the detrimental effect by the positive effect of immune-mediated toxicity itself. Approximately $20 \%$ of steroid-refractory irAEs required additional treatment, with the majority of those having received TNF- $\alpha$ inhibitors. Rapid resolution was observed in almost all cases with colitis. The relatively high rate of TNF- $\alpha$ or IL-6 inhibitors in our cohort is a result of our clinical judgment that early treatment or treatment in lower irAEs grades (e.g., grade 2) is preferable to prolonged treatment with high-dose corticosteroids.

A minority in our study cohort had underlying autoimmune toxicities, with only $35 \%$ of those experiencing a flare of their disease and $75 \%$ of those experiencing any kind of irAE. A previous study has reported a similar incidence of autoimmune disease exacerbation in 52 melanoma patients treated with anti-PD1 monotherapy [33]. In the same study, no patients with previous gastrointestinal disease flared upon exposure to ICIs: however, one patient in our study with inactive IBD started prophylactic treatment with a TNF- $\alpha$ inhibitor upon initiation of ICIs. This probably suggests that the pathogenesis of autoimmunity is heterogeneous with many disorders not relying on PD1 or CTLA4 pathway.

Noteworthy, $5 \%(n=9)$ of our patients experienced late-onset irAEs, defined as an irAE that occurred after treatment discontinuation, with endocrinological toxicities, skin toxicities and pneumonitis being the most frequent ones. These findings are comparable with a recent multicenter retrospective analysis reporting an estimated incidence of $5.7 \%$ of late onset irAEs and an identical pattern [34]. Although the incidence is low, late-onset irAEs can be difficult to manage and are definitely affecting patient's quality of life.

Our study has a number of limitations, including the retrospective, single-center design and low number of patients in some subgroups especially in the adjuvant setting, those with prior autoimmune diseases and those receiving steroids or other immunomodulating agents for irAEs. In addition, we must consider that since the cohort was not collected prospectively, under-reporting of mild toxicities cannot be excluded. On the other hand, it represents a relatively large homogenous cohort of exclusively melanoma patients treated with ICIs beyond clinical trials.

\section{Conclusions}

In conclusion, the appearance of irAEs in the non-adjuvant setting is undoubtedly associated with significant outcome benefit. We confirm previous reports on the low number of autoimmune disease flares with ICIs and support the non-significant impact of steroids and other immunomodulating agents, when administered early and for a short time, for irAE management on clinical outcome. Further elucidation of the predictive and prognostic role of irAEs in the prospective setting is warranted.

Supplementary Materials: The following are available online at https: / www.mdpi.com/article / 10.3390/cancers13122931/s1, Figure S1: Univariate Cox regression analysis for covariates in the 
adjuvant setting, Figure S2: Univariate Cox regression analysis for covariates in the unresectable/nonadjuvant setting.

Author Contributions: Conceptualization, J.M., R.D., P.F.C., F.D., M.A. and R.S.; formal analysis, P.F.C., F.D., M.A. and R.S.; data curation, M.M., M.A., R.S., M.J.B. and K.K.; writing—original draft preparation, J.M., P.F.C., F.D., R.S. and M.A.; writing-review and editing, J.M., R.D., P.F.C., F.D., M.A., R.S. M.J.B., M.P.L. and M.M.; visualization, P.F.C., F.D., R.S. and M.A. All authors have read and agreed to the published version of the manuscript.

Funding: This research did not receive any specific grant from funding agencies in the public, commercial, or not-for-profit sectors. The melanoma registry database of the Department of Dermatology Zurich is being partially supported by an unrestricted grant to the University of Zurich from Amgen, Novartis, BMS, MSD and Pierre Fabre. F.D. is supported by the Swiss Academy of Medical Sciences (SAMS) and G. and J. Bangerter-Rhyner Foundation (YTCR 22/19).

Institutional Review Board Statement: The study was conducted according to the guidelines of the Declaration of Helsinki and approved by the local Ethics Committee (KEK-ZH 2014-0193, in 2014).

Informed Consent Statement: Informed consent was obtained from all patients involved in the study.

Data Availability Statement: All data is available upon request. Written informed consent for the use and collection of data for the use in retrospective analysis was approved by the local ethics committee (MelProg Project KEK-ZH 2014-0193, KEK 2017-00494). The study was conducted in accordance with the Declaration of Helsinki.

Acknowledgments: We thank Isabell Pieper Scholz for her contribution to the melanoma registry database.

Conflicts of Interest: R.D. has intermittent, project focused consulting and/or advisory relationships with Novartis, Merck Sharp \& Dhome (MSD), Bristol-Myers Squibb (BMS), Roche, Amgen, Takeda, Pierre Fabre, Sun Pharma, Sanofi, Catalym, Second Genome, Regeneron, Alligator, T3 Pharma, MaxiVAX SA and touchIME outside the submitted work. M.P.L. receives project specific research funding, unrelated to the work presented here, from Roche, Novartis, Molecular Partners, and Oncobit AG. J.M. has intermittent project focused consultant or advisory relationships with Merck/Pfizer, Merck Sharp \& Dohme, Amgen, Novartis, Sanofi, Bristol Myers Squibb and Pierre Fabre and has received travel support from Ultrasun, L' oreal, Merck Sharp \& Dohme, Bristol Myers and Squibb und Pierre Fabre outside of the submitted work. F.D., R.S., M.A., M.M., K.K., M.J.B. and P.F.K. have declared no conflicts of interest.

\section{References}

1. Hodi, F.S.; Chiarion-Sileni, V.; Gonzalez, R.; Grob, J.J.; Rutkowski, P.; Cowey, C.L.; Lao, C.D.; Schadendorf, D.; Wagstaff, J.; Dummer, R.; et al. Nivolumab plus ipilimumab or nivolumab alone versus ipilimumab alone in advanced melanoma (checkmate 067): 4-year outcomes of a multicentre, randomised, phase 3 trial. Lancet Oncol. 2018, 19, 1480-1492. [CrossRef]

2. Schachter, J.; Ribas, A.; Long, G.V.; Arance, A.; Grob, J.J.; Mortier, L.; Daud, A.; Carlino, M.S.; McNeil, C.; Lotem, M.; et al. Pembrolizumab versus ipilimumab for advanced melanoma: Final overall survival results of a multicentre, randomised, openlabel phase 3 study (keynote-006). Lancet 2017, 390, 1853-1862. [CrossRef]

3. Eggermont, A.M.M.; Blank, C.U.; Mandala, M.; Long, G.V.; Atkinson, V.; Dalle, S.; Haydon, A.; Lichinitser, M.; Khattak, A.; Carlino, M.S.; et al. Adjuvant pembrolizumab versus placebo in resected stage iii melanoma. N. Engl. J. Med. 2018, 378, 1789-1801. [CrossRef] [PubMed]

4. Weber, J.; Mandala, M.; Del Vecchio, M.; Gogas, H.J.; Arance, A.M.; Cowey, C.L.; Dalle, S.; Schenker, M.; Chiarion-Sileni, V.; Marquez-Rodas, I.; et al. Adjuvant nivolumab versus ipilimumab in resected stage iii or iv melanoma. N. Engl. J. Med. 2017, 377, 1824-1835. [CrossRef] [PubMed]

5. Michot, J.M.; Bigenwald, C.; Champiat, S.; Collins, M.; Carbonnel, F.; Postel-Vinay, S.; Berdelou, A.; Varga, A.; Bahleda, R.; Hollebecque, A.; et al. Immune-related adverse events with immune checkpoint blockade: A comprehensive review. Eur. J. Cancer 2016, 54, 139-148. [CrossRef] [PubMed]

6. Baraibar, I.; Melero, I.; Ponz-Sarvise, M.; Castanon, E. Safety and tolerability of immune checkpoint inhibitors (pd-1 and pd-11) in cancer. Drug Saf. 2019, 42, 281-294. [CrossRef] [PubMed]

7. Wang, D.Y.; Salem, J.-E.; Cohen, J.V.; Chandra, S.; Menzer, C.; Ye, F.; Zhao, S.; Das, S.; Beckermann, K.E.; Ha, L.; et al. Fatal toxic effects associated with immune checkpoint inhibitors: A systematic review and meta-analysis. JAMA Oncol. 2018, 4, 1721-1728. [CrossRef] 
8. Shoushtari, A.N.; Friedman, C.F.; Navid-Azarbaijani, P.; Postow, M.A.; Callahan, M.K.; Momtaz, P.; Panageas, K.S.; Wolchok, J.D.; Chapman, P.B. Measuring toxic effects and time to treatment failure for nivolumab plus ipilimumab in melanoma. JAMA Oncol. 2018, 4, 98-101. [CrossRef] [PubMed]

9. Puzanov, I.; Diab, A.; Abdallah, K.; Bingham, C.O.; Brogdon, C.; Dadu, R.; Hamad, L.; Kim, S.; Lacouture, M.E.; LeBoeuf, N.R.; et al. Managing toxicities associated with immune checkpoint inhibitors: Consensus recommendations from the society for immunotherapy of cancer (sitc) toxicity management working group. J. Immunother. Cancer 2017, 5, 95. [CrossRef]

10. Stroud, C.R.G.; Hegde, A.; Cherry, C.; Naqash, A.R.; Sharma, N.; Addepalli, S.; Cherukuri, S.; Parent, T.; Hardin, J.; Walker, P. Tocilizumab for the management of immune mediated adverse events secondary to pd-1 blockade. J. Oncol. Pharm. Pract. 2017, 25, 551-557. [CrossRef]

11. Dimitriou, F.; Matter, A.V.; Mangana, J.; Urosevic-Maiwald, M.; Micaletto, S.; Braun, R.P.; French, L.E.; Dummer, R. Cytokine release syndrome during sequential treatment with immune checkpoint inhibitors and kinase inhibitors for metastatic melanoma. J. Immunother. (Hagerstown MD 1997) 2019, 42, 29-32. [CrossRef] [PubMed]

12. Weber, J.S.; Larkin, J.M.G.; Schadendorf, D.; Wolchok, J.D.; Wagstaff, J.; Dummer, R.; Hogg, D.; Guidoboni, M.; Sosman, J.A.; Chmielowski, B.; et al. Management of gastrointestinal (gi) toxicity associated with nivolumab (nivo) plus ipilimumab (ipi) or ipi alone in phase ii and iii trials in advanced melanoma (mel). J. Clin. Oncol. 2017, 35, 9523. [CrossRef]

13. Pan, E.Y.; Merl, M.Y.; Lin, K. The impact of corticosteroid use during anti-pd1 treatment. J. Oncol. Pharm. Pract. Off. Publ. Int. Soc. Oncol. Pharm. Pract. 2020, 26, 814-822. [CrossRef] [PubMed]

14. Das, S.; Johnson, D.B. Immune-related adverse events and anti-tumor efficacy of immune checkpoint inhibitors. J. Immunother. Cancer 2019, 7, 306. [CrossRef] [PubMed]

15. Ricciuti, B.; Genova, C.; De Giglio, A.; Bassanelli, M.; Dal Bello, M.G.; Metro, G.; Brambilla, M.; Baglivo, S.; Grossi, F.; Chiari, R. Impact of immune-related adverse events on survival in patients with advanced non-small cell lung cancer treated with nivolumab: Long-term outcomes from a multi-institutional analysis. J. Cancer Res. Clin. Oncol. 2019, 145, 479-485. [CrossRef] [PubMed]

16. Indini, A.; Di Guardo, L.; Cimminiello, C.; Prisciandaro, M.; Randon, G.; De Braud, F.; Del Vecchio, M. Immune-related adverse events correlate with improved survival in patients undergoing anti-pd1 immunotherapy for metastatic melanoma. J. Cancer Res. Clin. Oncol. 2019, 145, 511-521. [CrossRef]

17. Eggermont, A.M.M.; Kicinski, M.; Blank, C.U.; Mandala, M.; Long, G.V.; Atkinson, V.; Dalle, S.; Haydon, A.; Khattak, A.; Carlino, M.S.; et al. Association between immune-related adverse events and recurrence-free survival among patients with stage iii melanoma randomized to receive pembrolizumab or placebo: A secondary analysis of a randomized clinical trial. JAMA Oncol. 2020, 6, 519-527. [CrossRef]

18. Robert, C.; Hwu, W.-J.; Hamid, O.; Ribas, A.; Weber, J.S.; Daud, A.I.; Hodi, F.S.; Wolchok, J.D.; Mitchell, T.C.; Hersey, P.; et al. Long-term safety of pembrolizumab monotherapy and relationship with clinical outcome: A landmark analysis in patients with advanced melanoma. Eur. J. Cancer 2021, 144, 182-191. [CrossRef]

19. Common Terminology Criteria for Adverse Events (Ctcae). Available online: https:/ /ctep.cancer.gov/protocolDevelopment/ electronic_applications/ctc.htm (accessed on 11 June 2021).

20. Eisenhauer, E.A.; Therasse, P.; Bogaerts, J.; Schwartz, L.H.; Sargent, D.; Ford, R.; Dancey, J.; Arbuck, S.; Gwyther, S.; Mooney, M.; et al. New response evaluation criteria in solid tumours: Revised recist guideline (version 1.1). Eur. J. Cancer 2009, 45, $228-247$. [CrossRef]

21. Dupont, R.; Bérard, E.; Puisset, F.; Comont, T.; Delord, J.P.; Guimbaud, R.; Meyer, N.; Mazieres, J.; Alric, L. The prognostic impact of immune-related adverse events during anti-pd1 treatment in melanoma and non-small-cell lung cancer: A real-life retrospective study. Oncoimmunology 2020, 9, 1682383. [CrossRef]

22. Horvat, T.Z.; Adel, N.G.; Dang, T.O.; Momtaz, P.; Postow, M.A.; Callahan, M.K.; Carvajal, R.D.; Dickson, M.A.; D'Angelo, S.P.; Woo, K.M.; et al. Immune-related adverse events, need for systemic immunosuppression, and effects on survival and time to treatment failure in patients with melanoma treated with ipilimumab at memorial sloan kettering cancer center. J. Clin. Oncol. 2015, 33, 3193-3198. [CrossRef] [PubMed]

23. Suo, A.; Chan, Y.; Beaulieu, C.; Kong, S.; Cheung, W.Y.; Monzon, J.G.; Smylie, M.; Walker, J.; Morris, D.; Cheng, T. Anti-pd1induced immune-related adverse events and survival outcomes in advanced melanoma. Oncologist 2020, 25, 438-446. [CrossRef] [PubMed]

24. Sanlorenzo, M.; Vujic, I.; Daud, A.; Algazi, A.; Gubens, M.; Luna, S.A.; Lin, K.; Quaglino, P.; Rappersberger, K.; Ortiz-Urda, S. Pembrolizumab cutaneous adverse events and their association with disease progression. JAMA Dermatol. 2015, 151, 1206-1212. [CrossRef] [PubMed]

25. Dimitriou, F.; Long, G.V.; Menzies, A.M. Novel adjuvant options for cutaneous melanoma. Ann. Oncol. 2021, 32, 854-865. [CrossRef]

26. Martins, F.; Sofiya, L.; Sykiotis, G.P.; Lamine, F.; Maillard, M.; Fraga, M.; Shabafrouz, K.; Ribi, C.; Cairoli, A.; Guex-Crosier, Y.; et al. Adverse effects of immune-checkpoint inhibitors: Epidemiology, management and surveillance. Nat. Rev. Clin. Oncol. 2019, 16, 563-580. [CrossRef]

27. Teulings, H.E.; Limpens, J.; Jansen, S.N.; Zwinderman, A.H.; Reitsma, J.B.; Spuls, P.I.; Luiten, R.M. Vitiligo-like depigmentation in patients with stage iii-iv melanoma receiving immunotherapy and its association with survival: A systematic review and meta-analysis. J. Clin. Oncol. 2015, 33, 773-781. [CrossRef] [PubMed] 
28. Mangana, J.; Dimitriou, F.; Braun, R.; Ludwig, S.; Dummer, R.; Barysch, M.J. Single-center real-life experience with low-dose ipilimumab monotherapy in adjuvant setting for patients with stage iii melanoma. Melanoma Res. 2019, 29, 648-654. [CrossRef]

29. Lebbé, C.; Meyer, N.; Mortier, L.; Marquez-Rodas, I.; Robert, C.; Rutkowski, P.; Menzies, A.M.; Eigentler, T.; Ascierto, P.A.; Smylie, M.; et al. Evaluation of two dosing regimens for nivolumab in combination with ipilimumab in patients with advanced melanoma: Results from the phase iiib/iv checkmate 511 trial. J. Clin. Oncol. 2019, 37, 867-875. [CrossRef] [PubMed]

30. Bristol-Myers Squibb Announces Update on Checkmate-915 for Opdivo (Nivolumab) Plus Yervoy (Ipilimumab) versus Opdivo alone in Patients with Resected High-Risk Melanoma and pd-11 < 1\%. Available online: https://news.bms.com/ news / corporate-financial/2019/Bristol-Myers-Squibb-Announces-Update-on-CheckMate--915-for-Opdivo-nivolumabPlus-Yervoy-ipilimumab-Versus-Opdivo-Alone-in-Patients-with-Resected-High-Risk-Melanoma-and-PD-L1-1/default.aspx (accessed on 11 June 2021).

31. Petrelli, F.; Signorelli, D.; Ghidini, M.; Ghidini, A.; Pizzutilo, E.G.; Ruggieri, L.; Cabiddu, M.; Borgonovo, K.; Dognini, G.; Brighenti, M.; et al. Association of steroids use with survival in patients treated with immune checkpoint inhibitors: A systematic review and meta-analysis. Cancers 2020, 12, 546. [CrossRef] [PubMed]

32. Cortellini, A.; Tucci, M.; Adamo, V.; Stucci, L.S.; Russo, A.; Tanda, E.T.; Spagnolo, F.; Rastelli, F.; Bisonni, R.; Santini, D.; et al. Integrated analysis of concomitant medications and oncological outcomes from pd-1/pd-11 checkpoint inhibitors in clinical practice. J. Immunother. Cancer 2020, 8, e001361. [CrossRef]

33. Menzies, A.M.; Johnson, D.B.; Ramanujam, S.; Atkinson, V.G.; Wong, A.N.M.; Park, J.J.; McQuade, J.L.; Shoushtari, A.N.; Tsai, K.K.; Eroglu, Z.; et al. Anti-pd-1 therapy in patients with advanced melanoma and preexisting autoimmune disorders or major toxicity with ipilimumab. Ann. Oncol. 2017, 28, 368-376. [CrossRef] [PubMed]

34. Owen, C.N.; Bai, X.; Quah, T.; Lo, S.; Callaghan, S.; Martínez-Vila, C.; Bhave, P.; Reijers, I.; Gerard, C.L.; Aspelagh, S.; et al. 1138p delayed immune-related adverse events (iraes) on anti-pd1-based therapy. Ann. Oncol. 2020, 31, S761-S762. [CrossRef] 